\title{
Medical Image of the Week: Recurrent Sarcoidosis Resembling Malignancy
}

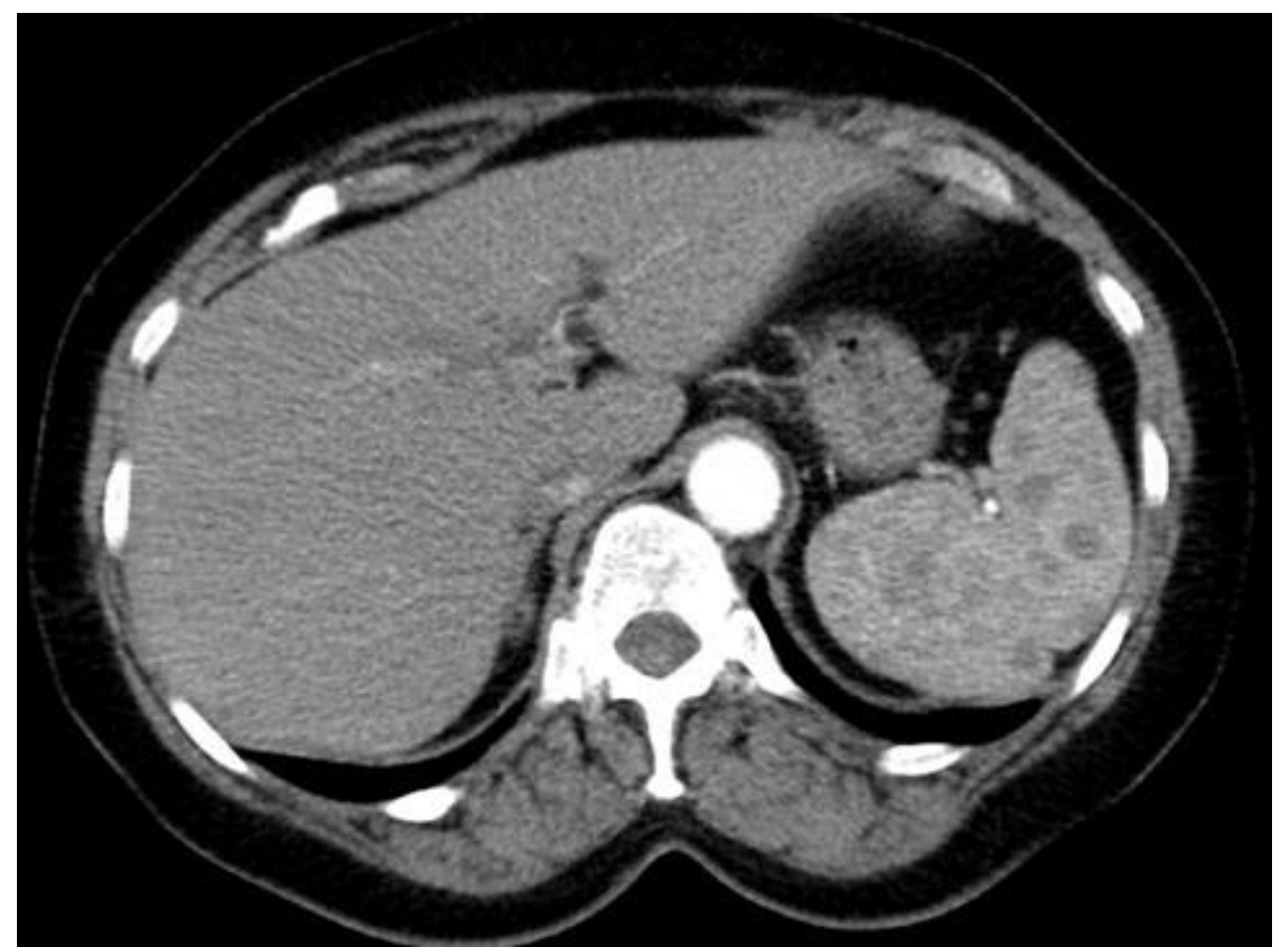

Figure 1. CT scan showing multiple splenic masses.

The patient is a 64-year-old, non-smoking, woman who presented with a dry cough for a year and had a history of sarcoidosis. Five years ago, she presented with cough, bilateral pulmonary nodules and mediastinal adenopathy. At that time by a fine needle aspiration (FNA) under endobronchial ultrasound biopsy (EBUS), revealing non caseating granulomatous inflammation. She had complete remission after she was treated with a course of steroids. Follow up chest CT showed remission of her disease. On this presentation, she was found to have incidental multiple splenic masses on a CT scan of the chest (Figure 1). Physical examination was normal, CBC and chemistry were normal, and she had negative tests for tuberculosis, rheumatologic disease, systemic sclerosis, and mixed connective tissue disease.

Pulmonary function tests were consistent with restrictive impairment and mild decrease in diffusion capacity for carbon monoxide. Positron Emission Tomography (PET scan) showed increased uptake corresponding to these lesions with no other areas of abnormal uptake in the body. CT-guided biopsy of splenic lesion showed non-caseating granulomas consistent with sarcoidosis. She was retreated with a course of steroids and a follow up CT scan of the abdomen showed resolution of the splenic masses.

Sarcoidosis is a systemic inflammatory disorder of unknown etiology characterized by non-caseating granulomas. It is thought to result from an exaggerated host cellular immune response to an unknown antigen. Although the lungs are involved in $90 \%$ of 
patients, other organs can be affected with the lungs or in isolation. Visceral involvement is usually asymptomatic and presents as hepatosplenomegaly on physical exam, or less often as isolated nodular splenic lesions suspicious of lymphoma. Rarely patients can have elevated liver enzymes from liver involvement or cytopenias from splenic enlargement (1).

Other differential diagnoses include infectious, neoplastic or benign conditions that may have similar morphology, such as tuberculosis, histoplasmosis, and lymphomas, hemangiomas and hamartomas. Diagnosis is usually confirmed by a biopsy and the disease responds to steroid treatment in over $70 \%$ of patients (2). Splenectomy is a therapeutic option in cases of steroids failure, severe hypersplenism, inability to exclude malignancy, or if risk of splenic rupture is high (3).

Isolated splenic sarcoidosis is a rare condition that can be the first presentation of sarcoidosis or a site of disease recurrence. It can mimic a variety of infectious, benign or malignant conditions especially when it presents with cytopenias due to hypersplenism, making biopsy necessary. Fortunately, splenic sarcoidosis responds to steroid treatment in most patients. Splenectomy can be considered in refractory cases.

Fuad Zeid MD¹, Hasan S. Yamin MD¹, Ahmed Amro MD², Fadi Alkhankan MD³, and Hani Alkhankan $\mathrm{MD}^{4}$

${ }^{1}$ Pulmonary and Critical Care and ${ }^{2}$ Internal Medicine

Marshall University

Huntington, WV USA

${ }^{3}$ Pulmonary and Critical Care

Mercy hospital/ St. Louis University

St. Louis, MO USA

${ }^{4}$ McLaren Oakland Pulmonary/Critical Care,

Pontiac, MI USA

\section{References}

Vakil A, MD, Upadhyay H, Sherani K, Cervellione K, Fein A. A case of splenic sarcoidosis: initial diagnosis and one-year symptom follow-up. Chest. 2014;145(3):210A [Abstract].

Calik M, Aygun M, Yesildag M, et al. Nodular splenic sarcoidosis: a rare case report and review of the literature. Chest. 2014;146(4):399A [Abstract].

Sharma OP, Vucinic V, James DG. Splenectomy in sarcoidosis: indications, complications, and long-term follow-up. Sarcoidosis Vasc Diffuse Lung Dis. 2002 Mar;19(1):66-70. [PubMed] 